\title{
Berufskrankheiten
}

\section{Die Crux mit dem Nachweis}

\section{Hauterkrankungen waren 2014 bei den Berufskrankheiten führend - allerdings nur bei der Zahl der bestätigten Fälle. Diese Indikation steht exemplarisch für den teils langen Weg bis zu einem Leistungsanspruch.}

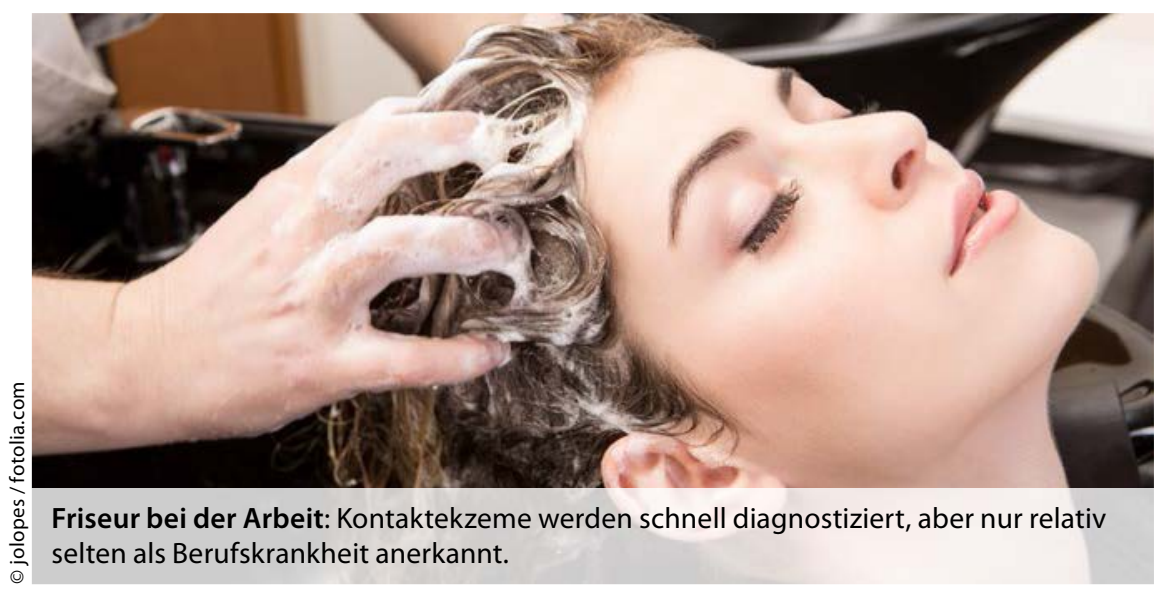

m Vergleich zum Vorjahr ist 2014 die Zahl der Anzeigen auf Verdacht einer Berufskrankheit leicht gestiegen und zwar um 0,2\% auf 71.589. Das geht aus dem aktuellen Bericht des Spitzenverbandes der Deutschen Gesetzlichen Unfallversicherung (DGUV) hervor. Bestätigt worden seien insgesamt 36.008 Fälle von Berufskrankheiten. Laut DGUV fehlte 2014 in 20.642 Fällen - obwohl nachgewiesen war, dass die Erkrankung beruflich verursacht wurde zur Anerkennung als Berufskrankheit im juristischen Sinne die Erfüllung der bei einigen Berufskrankheiten zusätzlich erforderlichen besonderen versicherungsrechtlichen Voraussetzungen.

\section{Großes Delta bei Hauterkrankungen} Die DGUV erklärt auch, was das bedeutet: Bei bestimmten Erkrankungen müssen für eine Anerkennung die Krankheiten zusätzlich zur Unterlassung aller Tätigkeiten geführt haben, die für die Entstehung, die Verschlimmerung oder das Wiederaufleben der Krankheit ursächlich waren oder sein können. Bei Hauterkrankungen (Berufskrankheitsnummer 5101) muss, so die DGUV, zudem das Tatbestandsmerkmal „schwere Krankheit oder wiederholte Rückfälligkeit“ erfüllt sein. „Dies bedeutet, dass trotz Vorliegen der ge zwischen versicherter Tätigkeit, schädigender Einwirkung und Erkrankung bei diesen Erkrankungen eine Anerkennung als Berufskrankheit im juristischen Sinne erst dann möglich ist, wenn diese zusätzlichen versicherungsrechtlichen Voraussetzungen erfüllt sind", heißt es in dem Jahresbericht.

Das führt zu der paradox anmutenden Situation, dass die Hauterkrankungen mit insgesamt 20.710 Fällen die Liste der beruflich verursachten Berufskrankheiten anführt - und zwar mit einem Anteil von $57,39 \%$. Da aber in 20.145 Fällen die weitergehenden versicherungsrechtlichen Anforderungen nicht erfüllt gewesen waren, wurde de facto nur in 565 Fällen die Hauterkrankung als Berufskrankheit auch tatsächlich anerkannt. genannten ursächlichen Zusammenhän-
Bessere Quoten bei Asbestose und Lärmschwerhörigkeit

Ganz anders sieht es bei der Lärmschwerhörigkeit (Nr. 2301) aus - sie liegt auf Platz zwei der im Jahre 2014 bestätigten Berufskrankheiten. Hier wurden nämlich alle bestätigten Fälle auch anerkannt. Auch bei der Asbestose (Nr. 4103; Platz drei mit 1.956 Fällen) und beim asbestbedingten Mesotheliom (Nr. 4105; Platz vier mit 1.040 Fällen) wurden alle Fälle anerkannt.

Die zum 1.1.2015 neue aufgenommene Nr. 5103 (Hautkrebs durch arbeitsbedingte UV-Strahlung) wird erst im nächsten DGUV-Jahresbericht berücksichtigt werden. Experten rechnen mit etwa 5.000 bis 7.000 Meldungen, was direkt Rang zwei ausmachen würde.

\section{Nicht immer folgt die Rente}

Wird eine Berufskrankheit anerkannt, so erfolgt nicht immer eine Rentenzahlung. „Bei den anerkannten Berufskrankheiten ohne Rentenzahlung werden vielfach Leistungen in anderer Form erbracht, zum Beispiel Heilbehandlung, Verletztengeld, Maßnahmen der beruflichen Rehabilitation oder Übergangsgeld“, so die DGUV. Im Berichtsjahr 2014 wurden den Angaben der DGUV zufolge insgesamt 75.179 Feststellungsverfahren abgeschlossen $-3,1 \%$ mehr als im Vorjahreszeitraum. Durch die höhere Zahl entschiedener Fälle sei es trotz gleichbleibend vieler Verdachtsanzeigen zu mehr Bestätigungen, Rentenbewilligungen und Ablehnungen als 2013 gekommen.

\section{Etwa jeder zweite Fall bestätigt}

In 36.754 Fällen sei der Verdacht auf das Vorliegen einer Berufskrankheit bestätigt worden, was einem Anteil von $48,9 \%$ entspreche. Unter diesen bestätigten Fällen hätten sich 16.112 anerkannte Berufskrankheiten im eigentlichen Sinne gefunden. In 5.155 dieser Fälle sei eine Rente, Abfindung oder Sterbegeld gezahlt worden. Matthias Wallenfels 